AperTO - Archivio Istituzionale Open Access dell'Università di Torino

\title{
Supports of Representations in the Cohen Class
}

\section{This is the author's manuscript}

Original Citation:

\section{Availability:}

This version is available http://hdl.handle.net/2318/94800

since 2016-10-19T01:10:21Z

Terms of use:

Open Access

Anyone can freely access the full text of works made available as "Open Access". Works made available under a Creative Commons license can be used according to the terms and conditions of said license. Use of all other works requires consent of the right holder (author or publisher) if not exempted from copyright protection by the applicable law. 
This is the author's final version of the contribution published as:

P. Boggiatto; C. Fernandez; A. Galbis. Supports of Representations in the Cohen Class. JOURNAL OF FOURIER ANALYSIS AND APPLICATIONS. Volume 17, Number 6 pp: 1180-1197.

When citing, please refer to the published version.

Link to this full text:

http://hdl.handle.net/2318/94800 


\title{
SUPPORTS OF REPRESENTATIONS IN THE COHEN CLASS
}

\author{
PAOLO BOGGIATTO, CARMEN FERNÁNDEZ AND ANTONIO GALBIS
}

\begin{abstract}
In this paper we consider a version of the uncertainty principle concerning limitations on the supports of time-frequency representations in the Cohen class. In particular we obtain different classes of kernels with the property that the corresponding representations of a non trivial signal can not be compactly supported. As an application of our results we show then that the Wigner function can never be solution of a partial differential equation with compactly supported datum.
\end{abstract}

\section{INTRODUCTION}

Quadratic structure of time-frequency representations is an intuitively reasonable assumption when we want to interpret a time-frequency representation as an energy distribution since the energy is a quadratic function of the signal. Moreover, quadratic expressions are often considered as ideal time-frequency representations because they do not depend on the choice of a window. In 1932 Wigner introduced the now called Wigner distribution in the setting of quantum mechanics and later Ville introduced it in signal theory. In 1933 Kirkwood came up with another quadratic distribution arguing that it was simpler to use than the Wigner distribution. Many variations were introduced, like the one of Rihaczek, Margeneau and Hille, Page, Choi and Williams, among others. In 1966 Cohen (see for instance [4]) gave a unified approach which can be formulated in a simple manner and proposed a general method for obtaining a family of joint time-frequency distributions. Following Gröchenig [12], a quadratic time-frequency representation $Q$ belongs to the Cohen's class if it is of the form

$$
Q(f)=\sigma * W(f)
$$

for some tempered distribution $\sigma$ called the kernel of $Q$, where $W(f)$ denotes the Wigner distribution of $f$. In particular, the action of a linear partial differential operator with constant coefficients on $W(f)$ can be written as a representation in the Cohen class whose kernel is a distribution supported at $\{0\}$. Some properties of the representation $Q$

2000 Mathematics Subject Classification. Primary 47G30. Secondary 35S05, 42B10, 44A35, 47B38.

Key words and phrases. Time-frequency representations; Cohen class; Wigner distribution; uncertainty principle. 
are reflected as simple constraints on the kernel $\sigma$. The relevance of the Cohen's class is due to the fact that every quadratic covariant timefrequency representation, satisfying some weak continuity assumption, belongs to this class [12, Theorem 4.5.1].

Recently the study of uncertainty principles for time-frequency representations has received the attention of several authors (see for instance $[3,7,8,15,16])$. It is usually assumed that any time-frequency representation should satisfy some appropriate version of the uncertainty principle. However, although it is well known that the Wigner distribution cannot be supported on a compact set (even on a set with finite Lebesgue measure [16, 22]), as far as we know there is no general result of this type for arbitrary representations in the Cohen class. In fact, very few is known more generally about uncertainty principles for the Cohen class [17].

The purpose of this paper is to propose some results in this direction. Our main interest will be to investigate under which conditions the support of a representation in the Cohen's class of a non-trivial signal cannot be compact. In some situations this conclusion is not hard to achieve. For instance, if the representation $Q$ satisfies the marginal property $([4,17])$ then the compactness of the support of $Q(f)$ would imply that the function $f$ and its Fourier transform would be compactly supported which forces the signal $f$ to be identically null. Another trivial situation is given by the action of the Cauchy-Riemann operator on the Wigner distribution. In this case $Q_{\sigma}(f)=\bar{\partial} W(f)$ being compactly supported would mean that $W(f)$ is a real valued holomorphic function outside of a disc, therefore constant, which gives $f=0$ for $f \in L^{2}\left(\mathbb{R}^{d}\right)$. But the same question for the Laplace operator is not so easy to answer. Let us observe that the study of the support of $P(D) W(f)$ gives information about the regularity properties of $W(f)$.

After a brief review of the basic notions that we shall need, we present our results in Sections 2 and 3, which essentially consist of a variety of cases where, under suitable hypothesis, the representation $Q_{\sigma}(f)$ of a non trivial signal $f$ cannot have compact support.

More precisely, in section 2 we show that this is the case under some specific conditions on the supports of the signal $f$ and the Fourier transform of the kernel $\sigma$ as well as for $f$ in suitable Gelfand-Shilov spaces. In section 3 we focus on the role of compactness on kernels and/or signals and that of hypo-ellipticity of the convolution operator with the kernels which enter in different ways for obtaining the desired results. As an application we show then that the Wigner function can never be solution of a partial differential equation with compactly supported datum.

For the background about time-frequency representations we refer to Gröchenig [12]. The reference for convolution operators and related topics is [14]. 


\section{Preliminary Results for Kernels in $\mathcal{S}^{\prime}\left(\mathbb{R}^{2 d}\right)$}

We start with some notation and definitions which will be used in the sequel.

Given a non-empty subset $A$ in $\mathbb{R}^{d}$ by acx $A$ we will denote the absolutely convex hull of $A$ that is

$$
\text { acx } A=\{\alpha x+\beta y: \alpha, \beta \in \mathbb{R},|\alpha|+|\beta| \leq 1, x, y \in A\} .
$$

Definiton 1. The cross Wigner distribution of $f, g \in L^{2}\left(\mathbb{R}^{d}\right)$ is

$$
W(f, g)(x, \omega)=\int_{\mathbb{R}^{d}} f\left(x+\frac{t}{2}\right) \overline{g\left(x-\frac{t}{2}\right)} e^{-2 \pi i \omega t} d t .
$$

When $f=g$ we write $W(f)=W(f, f)$. It happens that $W(f, g) \in$ $L^{2}\left(\mathbb{R}^{2 d}\right)$.

We will denote by $\mathcal{S}\left(\mathbb{R}^{d}\right)$ the Schwartz space of smooth and rapidly decreasing functions and by $\mathcal{S}^{\prime}\left(\mathbb{R}^{d}\right)$ its dual space. Using [12, 4.3.3], the cross Wigner distribution can be extended as a continuous map from $\mathcal{S}^{\prime}\left(\mathbb{R}^{d}\right) \times \mathcal{S}^{\prime}\left(\mathbb{R}^{d}\right)$ into $\mathcal{S}^{\prime}\left(\mathbb{R}^{2 d}\right)$ and it maps $\mathcal{S}\left(\mathbb{R}^{d}\right) \times \mathcal{S}\left(\mathbb{R}^{d}\right)$ into $\mathcal{S}\left(\mathbb{R}^{2 d}\right)$. This extension satisfies Moyal's formula, that is

$$
\langle W(f, g), W(\varphi, \psi)\rangle=\langle f, \varphi\rangle \overline{\langle g, \psi\rangle}
$$

where $\varphi, \psi \in \mathcal{S}\left(\mathbb{R}^{d}\right)$ and $\langle$,$\rangle means the extension of the inner product$ in $L^{2}\left(\mathbb{R}^{2 d}\right)$ to $\mathcal{S}^{\prime}\left(\mathbb{R}^{2 d}\right) \times \mathcal{S}\left(\mathbb{R}^{2 d}\right)$.

Definiton 2. Let $\sigma \in \mathcal{S}^{\prime}\left(\mathbb{R}^{2 d}\right)$ be given. The quadratic time-frequency representation $Q_{\sigma}$ is defined as

$$
Q_{\sigma}: \mathcal{S}\left(\mathbb{R}^{d}\right) \rightarrow \mathcal{S}^{\prime}\left(\mathbb{R}^{2 d}\right), \quad Q_{\sigma}(f)=\sigma * W(f) .
$$

In fact, $Q_{\sigma}(f)$ is a smooth function for every $f \in \mathcal{S}\left(\mathbb{R}^{d}\right)$. The Cohen's class consists precisely of the representations $Q_{\sigma}$ as defined in (1).

In the case that $\widehat{\sigma} \in L^{\infty}\left(\mathbb{R}^{2 d}\right)$ we have that $Q_{\sigma}$ is a continuous map

$$
Q_{\sigma}: L^{2}\left(\mathbb{R}^{d}\right) \rightarrow L^{2}\left(\mathbb{R}^{2 d}\right) .
$$

We say that $Q_{\sigma}$ satisfies Moyal's formula if

$$
\left\langle Q_{\sigma}(f), Q_{\sigma}(g)\right\rangle=|\langle f, g\rangle|^{2}
$$

for every $f, g \in L^{2}\left(\mathbb{R}^{d}\right)$. We want to mention that most of the results in this section also hold for arbitrary $f \in L^{2}\left(\mathbb{R}^{d}\right)$.

It is well-known that $W(f)$ cannot be compactly supported even in the case that $f$ belongs to $\mathcal{S}^{\prime}\left(\mathbb{R}^{d}\right) \backslash\{0\}$ (see [8]). The situation is completely different for representations in Cohen class, as the following example shows.

Example 3. $Q_{\sigma}(f)$ can be identically zero even if $\sigma \neq 0$ and $f \neq 0$. 
In fact,

$$
\widehat{W(f)}(\xi, \nu)=\int_{\mathbb{R}^{d}} f\left(t-\frac{\nu}{2}\right) \overline{f\left(t+\frac{\nu}{2}\right)} e^{-2 \pi i \xi t} d t .
$$

Hence, taking $f$ supported on $[-a, a]^{d}$ we have that $\widehat{W(f)}$ is supported on $\mathbb{R}^{d} \times[-2 a, 2 a]^{d}$. We now consider $\sigma \in \mathcal{S}^{\prime}\left(\mathbb{R}^{2 d}\right)$ such that the projection on the $\nu$ variables of $\operatorname{supp} \hat{\sigma}$ does not intersect $[-2 a, 2 a]^{d}$. Then

$$
Q_{\sigma}(f)=\mathcal{F}^{-1}(\hat{\sigma} \widehat{W(f)})=0 .
$$

Thus, it is natural to ask whether it is possible to find a tempered distribution $\sigma$ and a function $f$ in the Schwartz class $f$ such that $Q_{\sigma}(f)$ has non trivial compact support.

Theorem 4. Suppose that $\sigma \in \mathcal{S}^{\prime}\left(\mathbb{R}^{2 d}\right)$, and $f \in \mathcal{S}\left(\mathbb{R}^{d}\right)$, are such that $\operatorname{supp} \hat{\sigma} \neq \mathbb{R}^{2 d}$, or acx supp $\cap$ acx supp $\hat{f} \neq \mathbb{R}^{d}$, then the support of $Q_{\sigma}(f)$ is either empty or non compact.

Proof. Assume that $Q_{\sigma}(f)$ is compactly supported, by the Paley-WienerSchwartz Theorem $\widehat{W(f)} \cdot \hat{\sigma}$ is an analytic function. We have then two possibilities: either $\widehat{W(f)} \cdot \hat{\sigma}=0$ identically or its support is the whole $\mathbb{R}^{2 d}$. In case supp $\hat{\sigma} \neq \mathbb{R}^{2 d}$ we conclude that $\widehat{W(f)} \cdot \hat{\sigma}=0$, hence $Q_{\sigma}(f)=0$. Assume now that $\operatorname{supp} \hat{\sigma}=\mathbb{R}^{2 d}$. If $\widehat{W(f)} \cdot \hat{\sigma}=0$, then $W(f)=0$ from where $f \equiv 0$. If not, we necessarily have supp $\widehat{W(f)}=$ $\mathbb{R}^{2 d}$, hence from (2) we have

$$
\mathbb{R}^{d}=\Pi_{\nu} \operatorname{supp} \widehat{W(f)} \subset 2 \operatorname{acx} \operatorname{supp} f .
$$

From Parseval's formula we deduce that

$$
\mathbb{R}^{d}=\Pi_{\xi} \operatorname{supp} \widehat{W(f)} \subset 2 \operatorname{acx} \operatorname{supp} \hat{f} .
$$

From the previous proof we observe that in Theorem 4 the condition acx supp $f \cap \operatorname{acx} \operatorname{supp} \hat{f} \neq \mathbb{R}^{d}$ can actually be replaced by the slightly weaker condition $\overline{(\operatorname{supp} f-\operatorname{supp} f)} \cap \overline{(\operatorname{supp} \hat{f}-\operatorname{supp} \hat{f})} \neq \mathbb{R}^{d}$.

We observe also that in the special case that $\hat{\sigma}$ is compactly supported we have $Q_{\sigma}(f)(x, \omega)=\widehat{T}(x, \omega)$, where $T$ is the compactly supported distribution defined as $T_{\xi, \nu}=\widehat{W(f)}(-\xi, \nu) \hat{\sigma}_{\xi, \nu}$. Hence, $Q_{\sigma}(f)$ is the restriction of an entire function on $\mathbb{C}^{2 d}$.

Example 5. Let us fix two non zero windows $\varphi, \psi \in \mathcal{S}\left(\mathbb{R}^{d}\right)$ such that

$$
\overline{\operatorname{supp} \hat{\phi}-\operatorname{supp} \hat{\psi}} \neq \mathbb{R}^{d} \text { or } \overline{\operatorname{supp} \psi-\operatorname{supp} \phi} \neq \mathbb{R}^{d} \text {. }
$$

The generalized spectrogram, defined in [2] as

$$
\operatorname{Spec}_{\varphi, \psi}(f):=V_{\varphi} f \cdot \overline{V_{\psi} f}
$$


is a quadratic representation in the Cohen's class and the support of $\operatorname{Spec}_{\varphi, \psi}(f)$ is either trivial or non compact.

In fact, $\operatorname{Spec}_{\varphi, \psi}=Q_{\sigma}$ for $\sigma=W(\psi, \varphi)$. The Fourier transform of the kernel $\sigma$ can be expressed in terms of the ambiguity function associated to the windows $\varphi$ and $\psi$ as follows.

$$
\widehat{\sigma}(\xi, \nu)=A(\psi, \varphi)(-\nu, \xi)=\int_{\mathbb{R}^{d}} \psi\left(t-\frac{\nu}{2} \overline{\varphi\left(t+\frac{\nu}{2}\right)} e^{-2 \pi i t \xi} d t .\right.
$$

Then, from the hypothesis it is easy to check that $\operatorname{supp} \hat{\sigma} \neq \mathbb{R}^{2 d}$.

Remark 6. Note that (3) is satisfied for example when there exists a direction of $\mathbb{R}^{d}$ in which $\operatorname{supp} \phi$ and $\operatorname{supp} \psi($ or $\operatorname{supp} \hat{\phi}$ and $\operatorname{supp} \hat{\psi})$ are bounded.

From now on we restrict ourselves to the case $\operatorname{supp} \hat{\sigma}=\mathbb{R}^{2 d}$.

Proposition 7. Let $\sigma \in \mathcal{S}^{\prime}\left(\mathbb{R}^{2 d}\right)$ be such that $Q_{\sigma}: L^{2}\left(\mathbb{R}^{d}\right) \rightarrow L^{2}\left(\mathbb{R}^{2 d}\right)$ satisfies Moyal's relation. Then $Q_{\sigma}(f)$ cannot be compactly supported unless $f=0$.

Proof. First we note that

$$
\begin{aligned}
& \int_{\mathbb{R}^{2 d}} Q_{\sigma}(f)(s, t) \overline{Q_{\sigma}(f)(s-x, t-\omega)} d(s, t) \\
& =\int_{\mathbb{R}^{2 d}} Q_{\sigma}(f)(s, t) \overline{Q_{\sigma}\left(M_{\omega} T_{x} f\right)(s, t)} d(s, t) \\
& =\left|\int_{\mathbb{R}^{d}} f(t) \overline{M_{\omega} T_{x} f}(t) d t\right|^{2}=\left|V_{f} f(x, \omega)\right|^{2} .
\end{aligned}
$$

Therefore if $Q_{\sigma}(f)$ has compact support we get that $V_{f} f(x, \omega)$ has also compact support, which implies $f=0([16])$.

Proposition 8. Let $\sigma \in S^{\prime}\left(\mathbb{R}^{2 d}\right)$ be such that $\sigma * \mu=\delta$ for some $\mu \in \mathcal{E}^{\prime}\left(\mathbb{R}^{2 d}\right)$. If $f \in \mathcal{S}\left(\mathbb{R}^{2 d}\right)$ and $Q_{\sigma}(f)=\sigma * W(f)$ has compact support then $f=0$.

In particular, if $\sigma \in S^{\prime}\left(\mathbb{R}^{2 d}\right)$ satisfies the following conditions

a) $\hat{\sigma}$ has an analytic extension to $\mathbb{C}^{2 d}$

b) there exist constants $C, N, B>0$ such that

$$
|\hat{\sigma}(z)| \geq C(1+|z|)^{-N} e^{-B|\Im z|}
$$

then $f=0$ provided that $Q_{\sigma}(f)$ is compactly supported.

Proof. Clearly $\mu * Q_{\sigma}(f)=W(f)$ has compact support provided that $Q_{\sigma}(f)$ is compactly supported, but this implies $f \equiv 0$.

The second statement follows from the first one as from b) we have

$$
|1 / \hat{\sigma}(z)| \leq C^{-1}(1+|z|)^{N} e^{B|\Im z|}
$$

then from a) and the Paley-Wiener theorem it follows that $\mu=\mathcal{F}^{-1}[1 / \hat{\sigma}] \in$ $\mathcal{E}^{\prime}\left(\mathbb{R}^{2 d}\right)$ and it is clear that $\mu * \sigma=\delta$. 
To close this section we analyze the support of representations in the Cohen class when signals are taken is some subspaces of $\mathcal{S}\left(\mathbb{R}^{d}\right)$ which are invariant under Fourier transform and consisting in analytic functions. Hence, all non-zero functions in these classes satisfy supp $f=$ $\operatorname{supp} \hat{f}=\mathbb{R}^{d}$.

Definiton 9. (Gelfand-Shilov spaces) Let $\alpha, \beta \geq 0$ be real numbers, the Gelfand-Shilov space $\mathcal{S}_{\alpha}^{\beta}\left(\mathbb{R}^{d}\right)$ is defined as the set of all $C^{\infty}$ functions on $\mathbb{R}^{d}$ for which there exist positive constants $A_{1}, . ., A_{n}, B_{1}, . ., B_{n}, C$ such that

$$
\left\|x^{\lambda} \partial_{x}^{\mu} f(x)\right\|_{L^{\infty}\left(\mathbb{R}^{d}\right)} \leq C A^{\lambda} B^{\mu} \lambda^{\alpha \lambda} \mu^{\beta \mu}
$$

for every multi-indices $\lambda \in \mathbb{N}_{0}^{d}, \mu \in \mathbb{N}_{0}^{d}$ (where $A^{\lambda}=A_{1}^{\lambda_{1}} \cdot \ldots \cdot A_{n}^{\lambda_{n}}$, $\alpha \lambda=\left(\alpha \lambda_{1}, . ., \alpha \lambda_{n}\right)$, etc. $)$.

We collect those features of the Gelfand-Shilov spaces that will be needed later on, for a more complete treatment refer e.g. to [11] or [19]. Particularly important is the invariance of the Gelfand-Shilov spaces $\mathcal{S}_{\alpha}^{\alpha}\left(\mathbb{R}^{d}\right)$ under the Wigner transform (as well as under the STFT), more precisely (see [19] (Thm 3.8))

\section{Proposition 10.}

a) The space $\mathcal{S}_{\alpha}^{\beta}\left(\mathbb{R}^{d}\right)$ is non trivial if and only if $\alpha+\beta>1$ or if $\alpha+\beta=1$ and $\alpha, \beta \neq 0$. We shall suppose from now on to be in one of these two cases.

b) $\mathcal{S}_{\alpha}^{\beta}\left(\mathbb{R}^{d}\right)$ is dense in the Schwartz space $\mathcal{S}\left(\mathbb{R}^{d}\right)$.

c) The Fourier transform defines an isomorphism

$$
\mathcal{F}: S_{\alpha}^{\beta}\left(\mathbb{R}^{d}\right) \longrightarrow \mathcal{S}_{\beta}^{\alpha}\left(\mathbb{R}^{d}\right) .
$$

d) For $1 / 2 \leq \alpha<1$ the space $\mathcal{S}_{\alpha}^{\alpha}\left(\mathbb{R}^{d}\right)$ is contained in the space $\mathcal{A}\left(\mathbb{R}^{d}\right)$ of analytic functions on $\mathbb{R}^{d}$.

e) Let $f, g \in \mathcal{S}_{\alpha}^{\alpha}\left(\mathbb{R}^{d}\right), \alpha \geq 1 / 2$. Then $W(f, g) \in \mathcal{S}_{\alpha}^{\alpha}\left(\mathbb{R}^{2 d}\right)$.

The following proposition shows a case where restricting the signals to the Gelfand-Shilov spaces intervenes in the question of the support of Cohen class representations.

Proposition 11. Given $f \in \mathcal{S}_{\alpha}^{\alpha}\left(\mathbb{R}^{d}\right), 1 / 2 \leq \alpha<1$, and $\sigma \in \mathcal{S}^{\prime}\left(\mathbb{R}^{2 d}\right)$ such that supp $\hat{\sigma}$ has non empty interior, then if $\operatorname{supp} Q_{\sigma} f \neq \mathbb{R}^{2 d}$ we have $f \equiv 0$.

Proof. Since $f \in \mathcal{S}_{\alpha}^{\alpha}\left(\mathbb{R}^{d}\right)$, then, from Proposition $10(\mathrm{e}), W(f) \in$ $\mathcal{S}_{\alpha}^{\alpha}\left(\mathbb{R}^{2 d}\right)$. As $\alpha \in[1 / 2,1)$, from Proposition $10(\mathrm{~d})$, the function $W(f)$ is analytic and the same holds then for the convolution $Q_{\sigma}(f)=\sigma * W(f)$. Since $Q_{\sigma} f$ is analytic and $\operatorname{supp} Q_{\sigma} f \neq \mathbb{R}^{2 d}$ we have $Q_{\sigma}(f)=0$. Thus $\widehat{\sigma} \widehat{W(f)}=0$ and therefore $\widehat{W(f)}$ is identically zero on a set with nonempty interior. Since it is analytic, we conclude it is zero. 


\section{Compact supports and COHEn kernels}

We present in this section some results where the compactness of the Cohen kernel $\sigma$ and/or the signal $f$ as well as the hypoellipticity of the operator of convolution with $\sigma$ play, in different ways, a crucial role to establish limitations on the support of a representation $Q_{\sigma}(f)$ of a non trivial signal $f \neq 0$.

Proposition 12. Let $\sigma \in \mathcal{E}^{\prime}\left(\mathbb{R}^{2 d}\right)$ and $f \in \mathcal{S}\left(\mathbb{R}^{d}\right)$ be given. We assume that either $f$ or $\hat{f}$ is compactly supported. If $Q_{\sigma}(f)$ is supported in a set of finite Lebesgue measure, then either $f \equiv 0$ or $\sigma \equiv 0$.

Proof. We first assume that $f \in \mathcal{D}\left(\mathbb{R}^{d}\right)$. For almost every $x \in \mathbb{R}^{d}$ the set

$$
\left\{\omega \in \mathbb{R}^{d}: \omega \in \operatorname{supp} Q_{\sigma}(f)(x, \cdot)\right\}
$$

has finite Lebesgue measure. Since we are going to show that $Q_{\sigma}(f)(x, \cdot)$ is analytic, we will conclude that $Q_{\sigma}(f)(x, \cdot) \equiv 0$ for almost every $x$, which implies that $Q_{\sigma}(f) \equiv 0$ from where the conclusion follows.

To see that $Q_{\sigma}(f)(x, \cdot)$ is analytic, we observe that it is a $C^{\infty}$ function. Moreover, since $\sigma \in \mathcal{E}^{\prime}\left(\mathbb{R}^{2 d}\right)$ there are constants $C>0, N \in \mathbb{N}$, and a compact set $K \subset \mathbb{R}^{2 d}$ such that

$$
\left|\frac{\partial^{\beta}}{\partial \omega^{\beta}} Q_{\sigma}(f)(x, \omega)\right|=\left|\left\langle\sigma,\left(\frac{\partial^{\beta}}{\partial \omega^{\beta}} W(f)\right)(x-\cdot, \omega-\cdot)\right\rangle\right|
$$

is less than or equal to $C$ times the maximum, as $|\alpha| \leq N,|\gamma| \leq N$ and $(s, t) \in K$, of

$$
\left|\frac{\partial^{\alpha+\gamma}}{\partial s^{\alpha} \partial t^{\gamma}}\left(\frac{\partial^{\beta}}{\partial \omega^{\beta}} W(f)\right)(x-s, \omega-t)\right|=\left|\frac{\partial^{\beta}}{\partial \omega^{\beta}} \widehat{g_{\alpha, \gamma, s, t}}(\omega)\right|,
$$

where

$$
g_{\alpha, \gamma, s, t}(u)=\frac{\partial^{\alpha}}{\partial s^{\alpha}}\left(f\left(x-s+\frac{u}{2}\right) \overline{f\left(x-s-\frac{u}{2}\right)}\right) \cdot \frac{\partial^{\gamma}}{\partial t^{\gamma}}\left(e^{-2 \pi i t u}\right) .
$$

Since $f \in \mathcal{D}\left(\mathbb{R}^{d}\right)$ then there is a compact set $L \subset \mathbb{R}^{d}$ such that the family of functions $\left\{g_{\alpha, \gamma, s, t}\right\}$ is a bounded set in the Fréchet space $\mathcal{D}(L)$ of smooth functions supported on $L$. Consequently, the set $\left\{\widehat{g_{\alpha, \gamma, s, t}}\right\}$ of Fourier-Laplace transforms is a bounded set in $H\left(\mathbb{C}^{d}\right)$. In particular, for every $a>0$ and $\epsilon>0$ there is $A>0$ with the property that

$$
\left|\frac{\partial^{\beta}}{\partial \omega^{\beta}} \widehat{g_{\alpha, \gamma, s, t}}(\omega)\right| \leq A \epsilon^{|\beta|} \beta \text { ! }
$$

whenever $|\alpha| \leq N,|\gamma| \leq N,(s, t)$ runs on $K$ and $\omega \in[-a, a]^{d}$. It follows that $Q_{\sigma}(f)(x, \cdot)$ is an analytic function.

In the second case that $\hat{f} \in \mathcal{D}\left(\mathbb{R}^{d}\right)$ we can argue as before, having in mind that $W(\hat{f})(x, \omega)=W(f)(-\omega, x)$, to conclude that $Q_{\sigma}(f)(\cdot, \omega)$ is analytic for every $\omega \in \mathbb{R}^{d}$. 
We now restrict our attention to one-dimensional signals and we assume that $\sigma \in \mathcal{E}^{\prime}\left(\mathbb{R}^{2}\right)$. The argument used in Proposition 8 does not work the other way around, that is if $\sigma$ is a compactly supported distribution, $E \in \mathcal{D}^{\prime}\left(\mathbb{R}^{2 d}\right)$ and $\sigma * E=\delta$, we cannot conclude as in Proposition 8 that $Q_{\sigma}(f)$ is not compactly supported, since, except when $\sigma$ is a translation of the Dirac measure, $E \notin \mathcal{E}^{\prime}\left(\mathbb{R}^{2 d}\right)$. However if we assume that the convolution operator

$$
* \sigma: \mathcal{D}^{\prime}\left(\mathbb{R}^{2 d}\right) \rightarrow \mathcal{D}^{\prime}\left(\mathbb{R}^{2 d}\right)
$$

is hypoelliptic, meaning that $\sigma * F \in \mathcal{C}^{\infty}\left(\mathbb{R}^{2 d}\right)$ implies $F \in \mathcal{C}^{\infty}\left(\mathbb{R}^{2 d}\right)$, we will be able to conclude using the existence of compactly supported parametrices (see [14]).

Lemma 13. Let $\sigma \in \mathcal{E}^{\prime}\left(\mathbb{R}^{2}\right)$ be a compactly supported distribution such that the convolution operator $F \mapsto F * \sigma$ is hypoelliptic. We suppose that $f \in \mathcal{S}(\mathbb{R})$ and $Q_{\sigma}(f)$ is compactly supported. Then, for $h=|f|^{2}$ or $h=|\widehat{f}|^{2}$, there are test functions $\psi, \lambda \in \mathcal{D}(\mathbb{R})$ such that

$$
h=\lambda+h * \psi \text {. }
$$

Proof. Let $E \in \mathcal{D}^{\prime}\left(\mathbb{R}^{2}\right)$ be with singular support contained in $[-a, a]^{2}$ such that $E * \sigma=\delta$ ([14, Theorem 16.6.5]), fix $\psi \in \mathcal{D}(\mathbb{R})$ equals one on a neighborhood of $[-a, a]$ and define $\Psi:=\psi \otimes \psi$. Then

$$
(\Psi E) * \sigma=\delta+(\Psi-1) E * \sigma .
$$

Since $(\Psi-1) E$ is a $C^{\infty}$ function then we finally obtain

$$
\varphi:=(1-\Psi) E * \sigma \in \mathcal{D}\left(\mathbb{R}^{2}\right)
$$

and

$$
(\delta-\varphi) * W(f)=(\Psi E) * \sigma * W(f)=(\Psi E) * Q_{\sigma}(f)
$$

is a compactly supported $C^{\infty}$ function. Let $A>0$ be given so that $(\delta-\varphi) * W(f)$ vanishes outside $[-A, A]^{2}$ and fix $|x|>A$. Then

$$
\begin{aligned}
|f(x)|^{2} & =\int_{\mathbb{R}} W(f)(x, \xi) d \xi \\
& =\int_{\mathbb{R}}(W(f) * \varphi)(x, \xi) d \xi \\
& =\int_{\mathbb{R}}\left(\int_{\mathbb{R}^{2}} W(f)(x-s, \xi-t) \varphi(s, t) d s d t\right) d \xi \\
& =\int_{\mathbb{R}^{2}} \varphi(s, t)\left(\int_{\mathbb{R}} W(f)(x-s, \xi-t) d \xi\right) d s d t \\
& =\int_{\mathbb{R}^{2}} \varphi(s, t)|f|^{2}(x-s) d t d s=\left(|f|^{2} * \psi\right)(x)
\end{aligned}
$$


where

$$
\psi(s)=\int_{\mathbb{R}} \varphi(s, t) d t .
$$

Put $h:=|f|^{2}$. Then $\lambda:=h-h * \psi$ is compactly supported. We conclude that there are test functions $\psi, \lambda \in \mathcal{D}(\mathbb{R})$ such that

$$
h=\lambda+h * \psi \text {. }
$$

A similar decomposition holds for $h=\mid \widehat{f}^{2}$ (with different test functions $\lambda$ and $\psi)$.

At this point we need one more lemma.

Lemma 14. Let $h \in \mathcal{S}(\mathbb{R})$ and $\psi, \lambda \in \mathcal{D}(\mathbb{R})$ be given so that

$$
h=\lambda+h * \psi .
$$

Then there are $b>0$ and $C>0$ such that $\widehat{h}$ can be analytically extended to the band $|\Im z|<2 b$ and

$$
|h(x)| \leq C e^{-2 \pi b|x|}
$$

for every $x \in \mathbb{R}$.

Proof. In fact,

$$
\widehat{h}(x)=\widehat{\lambda}(x)+\widehat{h}(x) \widehat{\psi}(x)
$$

for all $x \in \mathbb{R}$. All the real singularities of

$$
G(z):=\frac{\widehat{\lambda}(z)}{1-\widehat{\psi}(z)}
$$

are removable. Moreover, $G$ has a finite number of singularities on each set $|\Im z|<a$, hence there is $b>0$ so that all the singularities of $G$ in the set $|\Im z|<2 b$ are real. We abuse of the notation and still denote by $G$ the holomorphic function on $|\Im z|<2 b$ obtained when we remove all the real singularities. Then $G$ is an extension of $\widehat{h}$. Now take $A>0$ such that both $\lambda$ and $\psi$ are supported in $[-A, A]$. Then, for every $N \in \mathbb{N}$ there is $C_{N}>0$ such that

$$
\max (\widehat{\lambda}(z), \widehat{\psi}(z)) \leq C_{N} e^{A|\Im z|}(1+|z|)^{-N} .
$$

For any fixed $x>0$ we have

$$
h(x)=\int_{-\infty}^{\infty} G(t) e^{2 \pi i t x} d t .
$$

Denote by $\gamma_{R}$ the rectangle with vertex $-R, R, R+i b,-R+i b$. Then

$$
\int_{\gamma_{R}} G(z) e^{2 \pi i z x} d z=0
$$


and, after estimating the integrals on the vertical segments as $R$ goes to infinity we deduce that

$$
h(x)=\int_{-\infty}^{\infty} \frac{\widehat{\lambda}(t+i b)}{1-\widehat{\psi}(t+i b)} e^{2 \pi i(t+i b) x} d t
$$

and

$$
|h(x)| \leq C e^{-2 \pi b x}
$$

for some constant $C>0$. In the case $x<0$ we can argument in the same way but with a rectangle in the lower half-plane and we finally get

$$
|h(x)| \leq C e^{-2 \pi b|x|}
$$

for every $x \in \mathbb{R}$.

Theorem 15. Let $\sigma \in \mathcal{E}^{\prime}\left(\mathbb{R}^{2}\right)$ be a compactly supported distribution such that the convolution operator $F \mapsto F * \sigma$ is hypoelliptic and let $f \in \mathcal{S}(\mathbb{R})$ be given. Then $Q_{\sigma}(f)$ is not compactly supported unless $f$ is identically zero.

Proof. We suppose that $f$ is not identically zero but $Q_{\sigma}(f)$ is compactly supported. According to Lemma 13, $|\hat{f}|^{2}$ satisfies the hypothesis of Lemma 14 , hence there is $b>0$ such that $|\widehat{f}(\omega)| \leq e^{-2 \pi b|\omega|}$ for all $\omega \in \mathbb{R}$. Then

$$
F(z):=\int_{\mathbb{R}} \widehat{f}(\omega) e^{2 \pi i \omega z} d \omega
$$

is a holomorphic function in the band $|\Im z|<b$, hence $f$ is a realanalytic function. On the other hand, by Lemma 13, there are test functions $\psi, \lambda \in \mathcal{D}(\mathbb{R})$ so that

$$
h=\lambda+h * \psi,
$$

where $h=|f|^{2}$. Since $h$ and also $h * \psi$ are real analytic functions we conclude that $\lambda$ is identically zero. Now we take Fourier transforms and deduce that $\widehat{h}=\widehat{h} \cdot \widehat{\varphi}$. Consequently $\widehat{\varphi}(\omega)=1$ whenever $\widehat{h} \neq 0$. Then it follows from the principle of analytic continuation that $\widehat{\varphi}(\omega)=1$ for all $\omega$, which is a contradiction.

Corollary 16. Let $f \in \mathcal{S}(\mathbb{R})$ be a non-zero function. Then $W(f)$ is not an harmonic function outside a compact set.

A natural problem would be to assume that the convolution operator is just surjective on $C^{\infty}\left(\mathbb{R}^{2 d}\right)$ and investigate if similar results still hold. We do not have at present a precise answer to this question.

Our next aim is to show that the hypothesis in the previous result can be weakened. In particular we want to show that the Wigner function can never be solution of a partial differential equation with compactly 
supported datum. The key is to give an appropriate extension of Lemmata 13 and 14 .

Lemma 17. Let $\sigma \in \mathcal{S}^{\prime}\left(\mathbb{R}^{2}\right)$ be a tempered distribution with the property that there are compactly supported distributions $\mu_{1}, \mu_{2} \in \mathcal{E}^{\prime}(\mathbb{R}) \backslash\{0\}$ such that the convolution operators $f \mapsto f * \mu_{j}(j=1,2)$ are hypoelliptic and $\widehat{\mu_{1}}(\xi)=\widehat{\sigma}(\xi, 0), \widehat{\mu_{2}}(\xi)=\widehat{\sigma}(0, \xi)$. We suppose that $f \in \mathcal{S}(\mathbb{R})$ and $Q_{\sigma}(f)$ is compactly supported. Then, for $h=|f|^{2}$ or $h=|\widehat{f}|^{2}$, there are test functions $\psi, \lambda \in \mathcal{D}(\mathbb{R})$ such that

$$
h=\lambda+h * \psi \text {. }
$$

Proof. We concentrate on the case $h=|f|^{2}$. By hypothesis,

$$
\chi:=\sigma * W(f) \in \mathcal{D}\left(\mathbb{R}^{2}\right) .
$$

Now put

$$
\varphi(x):=\int_{\mathbb{R}} \chi(x, y) d y, \quad \varphi \in \mathcal{D}(\mathbb{R}) .
$$

We claim that $\varphi=\mu_{1} *|f|^{2}$. In fact, using that

$$
\int_{\mathbb{R}} W(f)(x, y) d y=|f(x)|^{2}
$$

we have

$$
\widehat{\varphi}(\xi)=\widehat{\chi}(\xi, 0)=\widehat{\sigma}(\xi, 0) \widehat{W(f)}(\xi, 0)=\widehat{\mu_{1}}(\xi) \widehat{|f|^{2}}(\xi)
$$

and the claim is proved. Now, since $* \mu_{1}$ is an hypoelliptic operator there are $a>0$ and $E \in \mathcal{D}^{\prime}(\mathbb{R})$ with singular support contained in $[-a, a]$ such that $E * \mu_{1}=\delta$. Fix $\Phi \in \mathcal{D}(\mathbb{R})$ equals one on a neighborhood of $[-a, a]$. Then

$$
(\Phi E) * \mu_{1}=\delta-(1-\Phi) E * \mu_{1} .
$$

Since $(1-\Phi) E$ is a $C^{\infty}$ function then we have

$$
\psi:=(1-\Phi) E * \mu_{1} \in \mathcal{D}(\mathbb{R})
$$

and also

$$
\lambda:=(\Phi E) * \mu_{1} *|f|^{2}=(\Phi E) * \varphi \in \mathcal{D}(\mathbb{R}) .
$$

Finally

$$
|f|^{2}=\lambda+\psi *|f|^{2} \text {. }
$$

Partial integration with respect to $x$ gives the conclusion for $|\hat{f}|^{2}$

Now, we can proceed as in the proof of Theorem 15 to get the following.

Theorem 18. Let $\sigma \in \mathcal{S}^{\prime}\left(\mathbb{R}^{2}\right)$ be a tempered distribution with the property that there are compactly supported distributions $\mu_{1}, \mu_{2} \in \mathcal{E}^{\prime}(\mathbb{R})$ such that the convolution operators $f \mapsto f * \mu_{j}(j=1,2)$ are hypoelliptic and $\widehat{\mu_{1}}(\xi)=\widehat{\sigma}(\xi, 0)$, $\widehat{\mu_{2}}(\xi)=\widehat{\sigma}(0, \xi)$. Let $f \in \mathcal{S}(\mathbb{R})$ be given. Then $Q_{\sigma}(f)$ is not compactly supported unless $f$ is identically zero. 
Example 19. (1) Let $\sigma \in \mathcal{S}^{\prime}\left(\mathbb{R}^{2}\right)$ be a tempered distribution such that $Q_{\sigma}$ has the marginal property. Then $\sigma$ satisfies the hypothesis in Theorem 18 with $\mu_{1}=\mu_{2}=\delta$. In this case the conclusion of the Theorem was already known.

(2) Let $\mu \in \mathcal{E}^{\prime}(\mathbb{R})$ be a compactly supported distribution and define

$$
\sigma:=\mathcal{F}^{-1}(\widehat{\mu}(a x+b y+h(x y))) \in \mathcal{S}^{\prime}\left(\mathbb{R}^{2}\right),
$$

where $h$ is any continuous function of at most polynomial growth on $\mathbb{R}$ and vanishing at the origin. We suppose

(a) $* \mu$ is hypoelliptic and $a b \neq 0$

$$
\text { or }
$$

(b) $a=b=0$ and $\widehat{\mu}(0) \neq 0$.

Then $\sigma$ need not be compactly supported but satisfies the hypothesis of Theorem 18 .

(3) Let us assume that $\mu \in \mathcal{E}^{\prime}(\mathbb{R})$ is hypoelliptic and let $\lambda \in \mathbb{R}$ be such that $\hat{\mu}(\lambda) \neq 0$. Then, the generalized spectrogram with windows $\mu$ and $e^{2 \pi i \lambda t}$ is a distribution $Q_{\sigma}$ with the property that $Q_{\sigma}(f)$ is not compactly supported unless $f=0$. In fact,

$$
\sigma=W\left(\mu, e^{2 \pi i \lambda t}\right) \text { and } \hat{\sigma}(x, \omega)=e^{-2 \pi i \omega\left(\lambda+\frac{x}{2}\right)} \hat{\mu}(\lambda+x) .
$$

Hence

$$
\hat{\sigma}(0, \omega)=\hat{\mu}(\lambda) e^{-2 \pi i \omega \lambda}, \quad \hat{\sigma}(x, 0)=e^{-\pi i \lambda x} \hat{\mu}(\lambda+x)
$$

and we can apply Theorem 18.

We conclude with an application of our results to linear partial differential equations in time-frequency space. It is a consequence of Theorem 18 and, in our opinion, is the main result of this section. We prove that if $P\left(D_{x}, D_{y}\right)$ is a partial differential operator, then for $f \in \mathcal{S}(\mathbb{R})$, the Wigner function $W(f)$ can not be solution of the equation $P\left(D_{x}, D_{y}\right) u=v$ if the datum $v$ has compact support.

Theorem 20. Let $P\left(D_{x}, D_{y}\right)$ be a partial differential operator. Then, for $f \in \mathcal{S}(\mathbb{R}), P\left(D_{x}, D_{y}\right) W(f)$ is not compactly supported unless $f$ is identically zero.

Proof. In fact, let us assume that $P\left(D_{x}, D_{y}\right) W(f)$ is compactly supported. Now we factorize $P\left(D_{x}, D_{y}\right)=D_{x}^{n} D_{y}^{m} Q\left(D_{x}, D_{y}\right)$, where $m, n \in \mathbb{N}_{0}$ and $Q\left(0, D_{y}\right)$ and $Q\left(D_{x}, 0\right)$ are non-zero differential operators. Since $Q\left(D_{x}, D_{y}\right) W(f)$ is in the Schwartz class we get that also $Q\left(D_{x}, D_{y}\right) W(f)$ is compactly supported. Take $\sigma \in \mathcal{E}^{\prime}\left(\mathbb{R}^{2}\right) \backslash\{0\}$ with $\widehat{\sigma}=Q$ and define $\mu_{j}$ according to Theorem 18. It is obvious that the convolution operators $f \mapsto f * \mu_{j}(j=1,2)$ are hypoelliptic, since they are (non-zero) differential operators in one variable. Consequently $f$ is identically zero. 
It would be natural to ask if it is possible to find examples of kernels and signals for which the corresponding Cohen representation has non trivial compact support. At the present moment we do not have an answer to this question.

Acknowledgement : This paper was finished during a visit of the last two authors to the University of Torino. They want to thank the Department of Mathematics for their hospitality. The research of C. Fernández and A. Galbis was supported by MEC and FEDER, Project MTM2007-62643 and net MTM2006-26627-E.

\section{REFERENCES}

[1] M. Benedicks. On Fourier transforms of functions supported on sets of finite Lebesgue measure. J. Math. Anal. Appl. 106 (1), 180-183, 1985.

[2] P. Boggiatto,G. De Donno, A. Oliaro. Uncertainty principle, positivity and $L^{p_{-}}$ boundedness for generalized spectrograms. J. Math. Anal. Appl. 335 (1) , 93$112,2007$.

[3] A. Bonami, B. Demange. A survey on uncertainty principles related to quadratic forms. Collect. Math. Vol. Extra, 1-36, 2006.

[4] L. Cohen. Time-Frequency Distributions - A Review. Proc. of IEEE, 77(7), 941-981, 1989.

[5] L. Cohen. Time-Frequency Analysis. Prentice Hall Signal Proc. series, New Jersey, 1995.

[6] L. Cohen. The uncertainty principle for the short-time Fourier transform. Proc. Int. Soc. Opt. Eng. 22563, 80-90, 1995.

[7] B. Demange. Uncertainty principles for the ambiguity function. J. London Math. Soc. 72, 717-730, 2005.

[8] C. Fernández, A. Galbis, Annihilating Sets for the Short-Time Fourier Transform. Adv. Math. (2010), doi:10.1016/j.aim.2010.01.010

[9] G. B. Folland, A. Sitaram. The uncertainty principle: a mathematical survey. J. Fourier Anal. Appl., 3(3), 207-238, 1989.

[10] L. Galleani, L. Cohen. The Wigner distribution for classical systems. Physics Letters A, 302, 149-155, 2002.

[11] I.M. Gelfand, G.E. Shilov. Generalized functions, Vol.2. Academic Press, New York-London, 1968.

[12] K. Gröchenig. Foundations of Time-Frequency Analysis. Birkhäuser, Boston, 2001.

[13] K. Gröchenig, Uncertainty principles for time-frequency representations. Advances in Gabor analysis, Appl. Numer. Harmon. Anal., Birkhäuser Boston, Boston, MA, 11-30, 2003.

[14] L. Hörmander. The analysis of linear partial differential operators. II. Differential operators with constant coefficients. Springer-Verlag, Berlin, 2005.

[15] Ph. Jaming. Principe d'incertitude qualitatif et reconstruction de phase pour la transformée de Wigner. C. R. Acad. Sci. Paris Sér. I Math. 327 , (3), 249-254, 1998.

[16] A. J. A. Janssen. Proof of a conjecture on the supports of Wigner distributions. J. Fourier Anal. Appl., 4(6), 723-726, 1998.

[17] P. Korn. Some uncertainty principles for time-frequency transforms of the Cohen class. IEEE Trans. Signal Process. 53, 523-527, 2005.

[18] E. H. Lieb. Integral bounds for radar ambiguity functions and Wigner distributions. J. Math. Phys., 31(3), 594-599, 1990. 
[19] N. Teofanov, Ultradistributions and Time-Frequency Analysis. preprint ESI 1598 (2005).

[20] M. W. Wong, Weyl Transforms, Springer-Verlag, (1998).

[21] M. W. Wong. Wavelet Transform and Localization Operators. BirkhäuserVerlag, Basel, (2002).

[22] E. Wilczok. New Uncertainty Principles for the Continuous Gabor Transform and the Continuous Wavelet Transform. Documenta Math., (5) 201-226, 2000.

P. Boggiatto: Department of Mathematics, University of Torino, Via Carlo Alberto 10, 10123 Torino, Italy

E-mail address: paolo.boggiatto@unito.it

C. Fernández: Departamento de Análisis Matemático, Universidad de Valencia, Doctor Moliner 50, 46100 Burjasot (Valencia), Spain

E-mail address: Carmen.Fdez-Rosell@uv.es

A. Galbis: Departamento de Análisis Matemático, Universidad de VaLencia, Doctor Moliner 50, 46100 Burjasot (VAlencia), Spain

E-mail address: Antonio.Galbis@uv.es 